\title{
Short-time Chebyshev wave packet method for molecular photoionization
}

\author{
Zhaopeng Sun, Yujun Zheng \\ School of Physics, Shandong University, Jinan 250100, China
}

\begin{abstract}
In this letter we present the extended usage of short-time Chebyshev wave packet method in the laser induced molecular photoionizaton dynamics. In our extension, the polynomial expansion of the exponential in the time evolution operator, the Hamiltonian operator can act on the wave packet directly which neatly avoids the matrix diagonalization. This propagation scheme is of obvious advantages when the dynamical system has large Hamiltonian matrix. Computational simulations are performed for the calculation of photoelectronic distributions from intense short pulse ionization of $\mathrm{K}_{2}$ and NaI which represent the Born-Oppenheimer (BO) model and Non-BO one, respectively.
\end{abstract}

Keywords: Time-dependent wave packet, photoionization, Chebyshev polynomials.

\section{Introduction}

With the advent of ultrashort laser pulses, the investigations of molecular elementary processes in the time scale of femtoseconds and attoseconds become a reality. By analyzing the corresponding spectrum, some interesting phenomenons such as Autler-Townes splitting, electromagnetically induced transparency, the dynamic Stark shift, can be directly observed experimentally $[1,2,3,4,5]$. In time-resolved measurement, most of the studies have been concerned with femtosecond pump/probe ionization experiments due to its extremely sensitivity of signal detection $[6,7,8,9,10,11,12]$. The total ion yields can be detected using a second time-delayed ultrashort pulse. Transient absorption, fluorescence, photoionization or other signals which mimic the time evolution of the system can be mapped out in the time-resolved photoelectron spectrum.

Stimulated by the experimental advances, amount of theoretical efforts are rapidly growing to simulate and interpret these pump-probe spectroscopies. The real-time femtosecond ionization experiments in gas phase molecules have been

Email address: yzheng@sdu.edu.cn (Yujun Zheng)

Preprint submitted to Chem. Phys. Lett.

July 6, 2016

C) 2016. This manuscript version is made available under the Elsevier user license http://www.elsevier.com/open-access/userlicense/1.0/ 
analyzed in detail using either classical models [13] or quantum mechanics simulations $[14,15,16,17,18]$. In quantum community, a straightforward approach is to discrete the ionization continuum so that the calculation of an ionization signal for a fixed delay time directly scales with the number of discret energies chosen to describe the continuum. Under this consideration, Meier et al. presented a numerical method based on multiconfiguration time-dependent Hatree principle to calculate photoelectrons spectrum from multiphoton ionization of small molecules [16]. It gave an exact representation with fully account of nuclear dynamics. After that, the comparative study of the different ionization pathways in $\mathrm{K}_{2}$ dimer was introduced by Charron et al., which showed excellent agreement between experimental results and theoretical simulations. Their quantum mechanical treatments are based on wave packet dynamics using the Fourier-Split-Operator (SPO) formalism [19, 20]. In 2003, Sun et al. revealed the origin of A-T splitting phenomenon from vibrational coherent states of $\mathrm{Na}_{2}$ molecule using this wave packet method [18]. However, all the theoretical frameworks mentioned above need to diagonalize the Hamiltonian matrix and they are much time consuming to treat the ionization continuum, and more effective numerical methods are in demand.

In this letter, we present the extended usage of the Short-time Chebyshev wave packet method ( $\mathrm{SCH}$ ) to calculate kinetic energy distributions of photoelectrons in the case of interaction with intense laser pulses. The SCH method was widely used in time-dependent quantum mechanical calculations such as molecular reactive scattering [22, 23, 24, 25], photodissociation processes [26, 27, 28] and optimal control dynamics [29]. However, when the system has time-dependent Hamiltonian, this algorithm tends to be inefficiently for dealing with single or fewer electronic states. Only fewer theoretical studies that propagate the wave packet using Chebyshev propagator have been reported $[29,30,31]$.

In photoionization dynamics, when discretize the ionization continuum into a band of quasi-continuum levels, the Hamiltonian matrix will be rather large. For traditional SPO method the numerical calculations are taking the extremely amount of CPU time. Thus, in this case, we can also take numerical simulations by employing $\mathrm{SCH}$, but we need to normalized Hamiltonian matrix at each time step and suppose the Hamiltonian is time independent in the time interval. Based on our considering, the adiabatic $\mathrm{K}_{2}$ molecule and nonadiabatic $\mathrm{NaI}$ molecule are taken as examples to test the accuracy and efficiency of this method.

The structure of this letter is as follows. In Sec. 2 we describe the theoretical framework of the title method. Results of the dynamics calculation and compared with other theoretical and experimental results are shown in Sec. 3. The paper ends with a short conclusion in Sec. 4.

\section{Theory}

In this section we brief describe the extension of $\mathrm{SCH}$. We consider the model of the multiphoton excitation and ionization of molecules by strong femtosecond 
pulses $[32,33]$. Within this model, the field-free molecular Hamiltonian $\hat{H}_{0}$ and interaction term $\hat{H}^{\prime}$ can be written as

$$
\begin{aligned}
\hat{H}_{0}= & \sum_{n, n^{\prime}=1}^{N}|n\rangle H_{n, n^{\prime}}\left\langle n^{\prime}\left|+\int d E\right| E\right\rangle\left(H_{I}+E\right)\langle E|, \\
\hat{H}^{\prime}= & \sum_{n \neq n^{\prime}=1}^{N}|n\rangle W_{n, n^{\prime}}\left\langle n^{\prime}\right|+ \\
& \sum_{n=1}^{N} \int\left(d E|n\rangle W_{n E}\langle E|+| E\rangle W_{n E}\langle n|\right),
\end{aligned}
$$

where $|n\rangle$ denotes electronic state of the neutral molecule, and $|E\rangle$ stands for orthonormal scattering state of the photoelectronic kinetic energy $E$ for interaction with an external laser field. $H_{n, n^{\prime}}$ are the Hamiltonians of the neutral molecule, the diagonal terms represent the coupling between the core and electrons and $H_{I}$ is the Hamiltonian for the ionization state. For the field-molecule interaction Hamiltonian $H^{\prime}, W_{i, j}=\mu_{i, j} \xi(t)$, where $\mu_{i, j}$ is the transition dipole moment (TDM) between electronic states and $\xi(t)$ denotes the time-dependent electronic field.

This model allows us to discrete the ionization continuum into a band of quasicontinuum levels in terms of electron eigenstates $\left|E_{i}\right\rangle$. Then, the total wave function (for 1-D case) can be written as

$$
|\Psi(R)\rangle=\sum_{n=1}^{N} \psi_{n}^{\mathrm{neu}}(R)|n\rangle+\sum_{i=1}^{N_{i}} \psi_{i}^{\text {ion }}(R)\left|E_{i}\right\rangle .
$$

For the time-dependent quantum calculation of the photoionization dynamics, the key step is to propagate the wave packet with time. By employing the Chebyshev polynomial, the propagator can be expressed as [21]

$$
\begin{aligned}
\psi(t+\Delta t)= & e^{-i H(t) \Delta t} \psi(t) \\
\approx & e^{-i \bar{E} \Delta t}\left(2-\delta_{n 0}\right) \sum_{n} J_{n}(\Delta E \cdot \Delta t) \times \\
& \Phi_{n}\left(-i H_{\text {norm }}\right) \psi(t) .
\end{aligned}
$$

The spectral half-width and mean $\bar{E}=\left(E_{\max }+E_{\min }\right) / 2, \Delta E=\left(E_{\max }-E_{\min }\right) / 2$ can be obtained from the maximum and minimum energy $\left(E_{\max }=V_{\max }+T_{\max }\right.$, $\left.E_{\min }=V_{\min }\right) . J_{n}$ is the Bessel function of the first kind, which converges to zero exponentially with $n$ increasing. The complex Chebyshev polynomials are generated by the three-term recurrence relation:

$$
\phi_{n+1}=-2 i H_{\mathrm{norm}} \phi_{n}+\phi_{n-1},
$$

with $\phi_{n}=\Phi_{n}\left(-i H_{\text {norm }}\right) \psi(t)$. The recurrence is started with $\phi_{0}=\psi(t)$ and $\phi_{1}=-i H_{\text {norm }} \psi(t)$. This could mean that the normalized Hamiltonian can act 
on the wave packet directly by multiplication in the coordinate space. The $H_{\text {norm }} \phi$ can, for the photoionization model, be written as

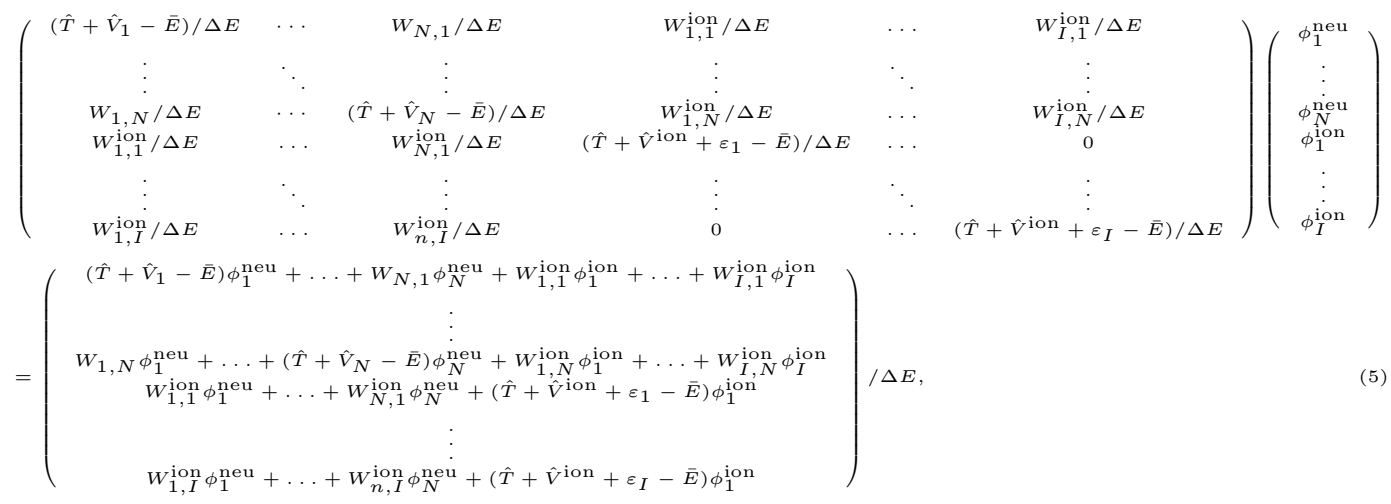

where $\varepsilon_{i}=(i-1) \Delta \varepsilon(i=1,2, \ldots, I)$ is the energy of the emitted photoelectron. $\phi_{n}^{\text {neu }}$ denote the different electronic states of the neutral molecule, and $\phi_{i}^{\text {ion }}$ stand for the ionization continuum. $\hat{T}=-\hbar^{2} \nabla^{2} / 2 \mu$ is the kinetic energy operator (KEO) which is nonlocal in the coordinate space. In our calculation, the fast fourier transformation (FFT) is used to calculate the action of the KEO on the propagating wave packet [34]. The time interval can be chosen for short enough to ensure the convergency of the wave packet since the Hamiltonian can be supposed to be time-independent at each time step. It can be properly broadened along with the wavelength of the laser pulse increasing. The potential energy operator $\hat{V}$ and coupled matrix $W_{i j}$ are diagonal in the coordinate space. Thus, they can act on the wave packet directly by multiplication in the coordinate space.

The time-dependent population of neutral electronic state can be written as

$$
P_{n}(t)=\int d R\left|\psi_{n}^{\text {neu }}(R, t)\right|^{2},
$$

and photoelectron spectrum can be obtained via

$$
P\left(\varepsilon_{i}\right)=\lim _{t \rightarrow \infty} \int d R\left|\psi_{i}^{\text {ion }}(R, t)\right|^{2} .
$$

\section{Results and Discussion}

\subsection{Adiabatic model: Laser induced Autler-Townes (AT) splitting in $K_{2}$}

Following the works by Nicole [12] and Yao [35] which had studied using SPO method, the same four state model is used in our simulation. The schematic diagram is shown in Fig 1 . The strong laser pulse applied to the $\mathrm{K}_{2}$ molecule results in transition from ground neutral state ${ }^{1} \Sigma_{g}^{+}$to the ionic state ${ }^{2} \Sigma_{g}^{+}$by a three-photon process. A center wavelength of $785 \mathrm{~nm}$ square pulse with intensity of $5 \times 10^{11} \mathrm{~W} / \mathrm{cm}^{2}$ and duration of $30 \mathrm{fs}$ is used in our works. We assume that 


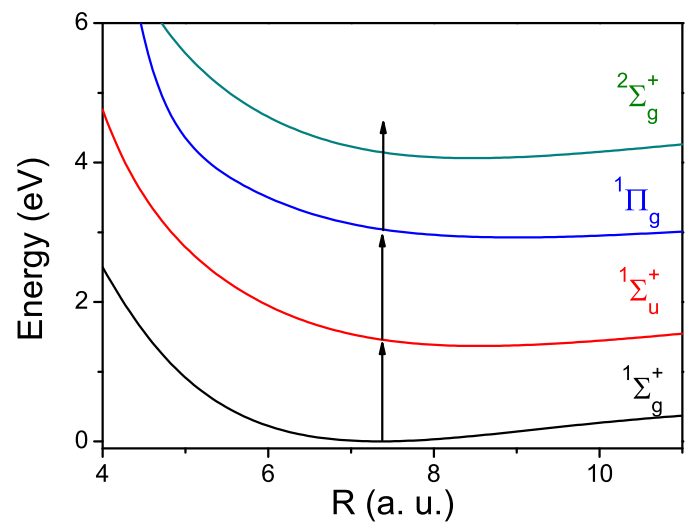

Figure 1: Excitation scheme for the three-photon ionization of $\mathrm{K}_{2}$. The model include three neutral electronic states $\left({ }^{1} \Sigma_{g}^{+},{ }^{1} \Sigma_{u}^{+},{ }^{1} \Pi_{g}\right)$ and the ionic ground state $\left({ }^{2} \Sigma_{g}^{+}\right)$

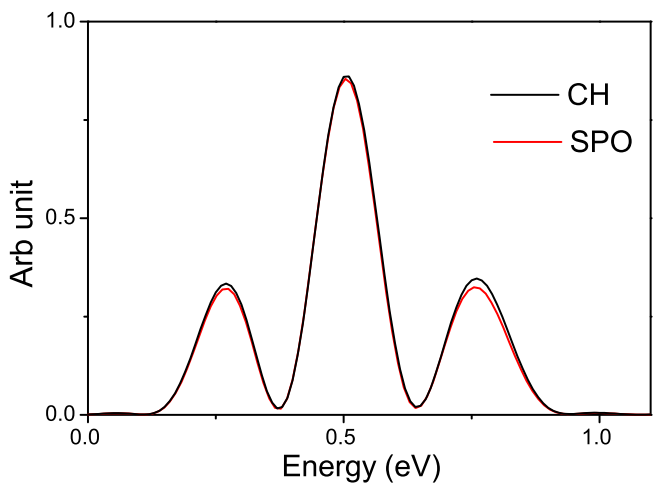

Figure 2: Energy dependence of the photoelectron spectrum of $\mathrm{K}_{2}$ molecule. The red line denotes the SPO result.

the system is initially populated at it ground vibrational state of the ground electronic state. The Condon approximation [36] is employed in our numerical calculations.

Fig 2 displays AT splitting spectroscopy of the photoelectrons created by an intense laser pulse. This phenomena results from rapid Rabi oscillations between different vibrational coherent states of each potential energy curves during field-molecule interaction [18]. Thus, the maximal number of the splitting peaks coincide with the amount of the field coupling states. As the figure shows, by 120 discrete values for representing the ionic state, our result consists with the result of SPO method. A slight difference of the center peak may 


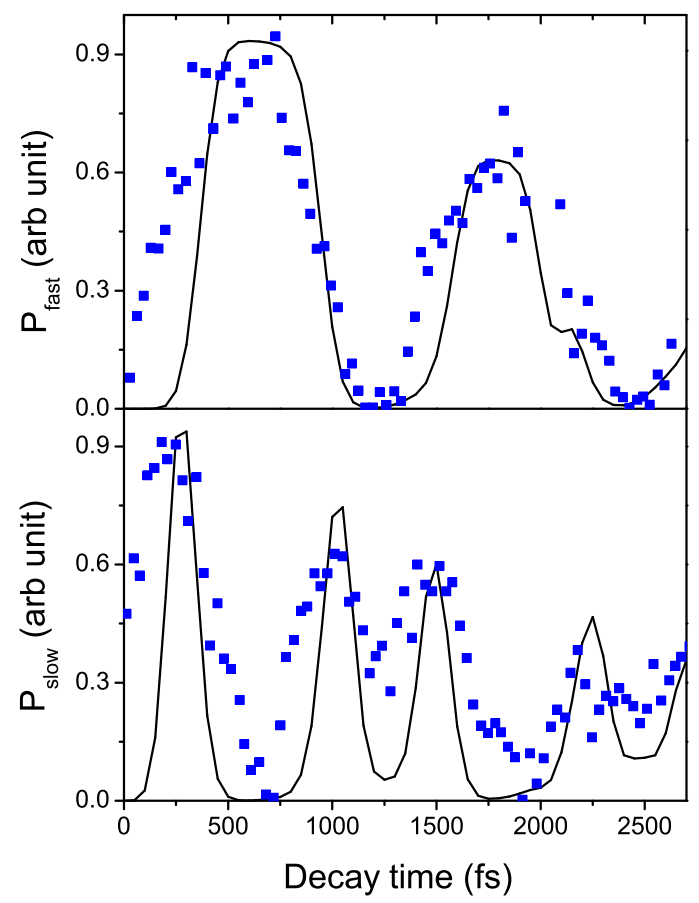

Figure 3: The fast and slow electron signals (Black solid line) as a function of pump-probe delay time. The blue squares are the experimental data from Ref. [37]. 
stem from the approximation of the SPO scheme. Because $\hat{T}$ and $\hat{V}$ are not commuted, the error will be proportional to the commutator $[\hat{T}, \hat{V}]$ and time interval $\Delta t$. As far as we know, the computational cost of each method is mainly determined by the numbers of FFT and by the diagonalization of the dipole coupling matrices at each time step. However, with the increase of the rank of the Hamiltonian matrix, the former will occupy a leading position and will determine the computation time. In this case, the SCH method shows its estimation capability and advantage.

\subsection{Diabatic model: Femtosecond photoionization of NaI molecule}

In this section we take the photoionization on diabatic potential of $\mathrm{NaI}$ as a further application of our proposed method. This system has already been extensively studied both from theoretically [38, 39, 40, 41, 42] and experimentally $[43,44]$. In our calculation, the diabatic potentials and TDMs are obtained from Ref. [39], and all the numerical aspects for dynamics follow Ref. [42]. Since the diabatc potentials also have non-diagonal terms, we can do the similar treatment as the TDM terms.

The ion signal of fast photoelectrons $(0.25 \mathrm{eV}-0.5 \mathrm{eV})$ and slow photoelectrons $(0 \mathrm{eV}-0.06 \mathrm{eV})$ as a function of decay time are shown in Fig 3. It can be seen from the figure that our theoretical result correctly predict position of peaks in the experimental signals [37]. The peaks' heights are reduced with decay time which can be attributed to the influence of nonadiabatic effects on the movement of wave packets.

\section{Conclusion}

In this letter, we presented the extension of the SCH wave packet method in the case of simulations of the kinetic energy distributions of electrons from molecular multiphoton ionization with laser pulses. In the photoionization dynamics, the molecular electronic continuum can be treated as a band of discrete electronics states, this leads the Hamiltonian matrix to be rather large. With the extended framework of $\mathrm{SCH}$, the Hamiltonian matrix can act on the wave packet directly which avoids the matrix diagonalization at each time step and leads to significant savings in computer time. As examples, the adiabatic $\mathrm{K}_{2}$ and nonadiabatic $\mathrm{NaI}$ are employed to show the efficiency and accuracy of this method. Our calculations are in well agreement with other theoretical and experimental results, respectively.

\section{Acknowledgements}

This work was supported by the National Science Foundation of China (Grant NOs. 91021009, 21073110)and partial supported by BNLMS fund project (20140101).

[1] S. H. Autler, C. H. Townes, Phys. Rev 100 (1955) 703. 
[2] J. Qi, G. Lazarov, X. wang, L. Li, L. M. Narducci, A. M. Lyyra, F. C. Dpano, Phys. Rev. Lett. 83 (1999) 288.

[3] S. Babin, U. Hinze, E. Tiemann, B. Wellegehausen, Opt. Lett. 21 (1996) 1186 .

[4] A. V. Sokolov, D. R. walker, D. D. Yavuz, G. Y. Yin, S. E. Harris, Phys. Rev. Lett. 87 (2001) 033402.

[5] R. Neuhauser, H. J. Neusser, J. Chem. Phys. 103 (1995) 5362.

[6] H. Ruppe, S. Rutz, E. Schreiber, L. Wöste, Chem. Phys. Lett 257 (1996) 356.

[7] T. Baumert, J. L. Herek, A. H. Zewail, J. Chem. Phys 99 (1993) 4430.

[8] V. Blanchet, M. A. Bouchene, O. Cabrol, B. Girard, Chem. Phys. Lett 233 (1995) 491.

[9] G. Rodriguez, J. G. Eden, Chem. Phys. Lett 205 (1993) 371.

[10] G. Rodriguez, P. C. John, J. G. Eden, J. Chem. Phys 103 (1995) 10473.

[11] H. Schwoerer, R. Pausch, M. Heid, V. Engel, W. Kiefer, J. Chem. Phys 107 (1997) 9749.

[12] C. Nicole, M. A. Bouchene, C. Meier, S. Magnier, E. Schreiber, B. Girard, J. Chem. Phys 111 (1999) 7857.

[13] J. A. Yeazell, M. Mallalieu, J. Parker, C. R. Stroud, Phys. Rev. A. 40 (1989) 5040.

[14] V. Engel, Chem. Phys. Lett. 178 (1991) 130.

[15] V. Engel, H. Metiu, Chem. Phys. Lett. 155 (1989) 77.

[16] C. Meier, V. Engel, U. Manthe, J. Chem. Phys 109 (1998) 36.

[17] M. Seel, W. Domcke, J. Chem. Phys 95 (1991) 7806.

[18] Z. Sun, N. Lou, Phys. Rev. Lett. 91 (2003) 023002.

[19] D. Feit, J. A. Fleck, A. Steiger, Comput. Phys. Commun. 47 (1982) 412.

[20] C. Meier, V. Engel, Chem. Phys. Lett. 212 (1993) 691.

[21] R. Kosloff, J. Chem. Phys 81 (1984) 3967.

[22] G. G. Balint-Kurti, A. L. Gonzalez, E. M. Goldfield, S. K. Gray, Faraday Discussions 110 (1998) 169.

[23] S. K. Gray, G. C. Balint-Kurti, J. Chem. Phys 108 (1998) 950. 
[24] S. Y. Lin, H. Guo, J. Phys. Chem. A 108 (2004) 2141.

[25] Z. Sun, C. F. Zhang, S. Y. Lin, Y. J. Zheng, Q. T. Meng, W. S. Bian, J. Chem. Phys 139 (2013) 014306.

[26] G. C. Balint-Kurti, S. P. Mort, C. C. Marston, Comput. Phys. Commun 74 (1993) 289.

[27] G. C. Balint-Kurti, L. Füsti-Molnár, A. Brown, Phys. Chem. Chem. Phys. 3 (2001) 702 .

[28] M. N. Daud, G. C. Balint-Kurti, Chin. Phys. Lett 26 (2009) 073302.

[29] M. Ndong, H. Tal-Ezer, R. Kosloff, C. P. Koch, J. Chem. Phys 130 (2009) 124108.

[30] Z. Sun, Y. Zheng, J. Phys. Chem. A. 119 (12) (2015) 2982.

[31] Z. Sun, C. Yang, Y. Zheng, J. Chem. Phys. 143 (2015) 224309.

[32] M. Seel, W. Domcke, Chem. Phys. 151 (1991) 59.

[33] M. Seel, W. Domcke, J. Chem. Phys. 95 (1991) 7806.

[34] D. Kosloff, R. Kosloff, J. Comput. Phys. 52 (1983) 35.

[35] H. Yao, Y. Zheng, Phys. Chem. Chem. Phys 13 (2011) 8900.

[36] M. Braun, C. Meier, V. Engel, J. Chem. Phys. 105 (1996) 530.

[37] C. Jouvet, S. Martrenchard, D. Solgadi, C. Dedonder-Lardeux, J. Phys. Chem. A 101 (1997) 2555.

[38] V. Engel, M. H, Chem. Phys. Lett 155 (1989) 77.

[39] M. Braun, C. Meier, V. Engel, J. Chem. Phys 105 (1996) 530.

[40] P. Marquetand, V. Engel, Chem. Phys. Lett 407 (2005) 471.

[41] Y. Han, K. Yuan, W. Hu, S. Cong, J. Chem. Phys 130 (2009) 044308.

[42] H. Yao, S. Y. Lin, Y. Zheng, J. Theo. Comput. Chem 10 (2011) 509.

[43] T. S. Rose, M. J. Rosker, A. H. Zewail, J. Chem. Phys. 88 (1988) 6672.

[44] M. J. Rosker, T. S. Rose, A. H. Zewail, Chem. Phys. Lett 146 (1988) 175. 


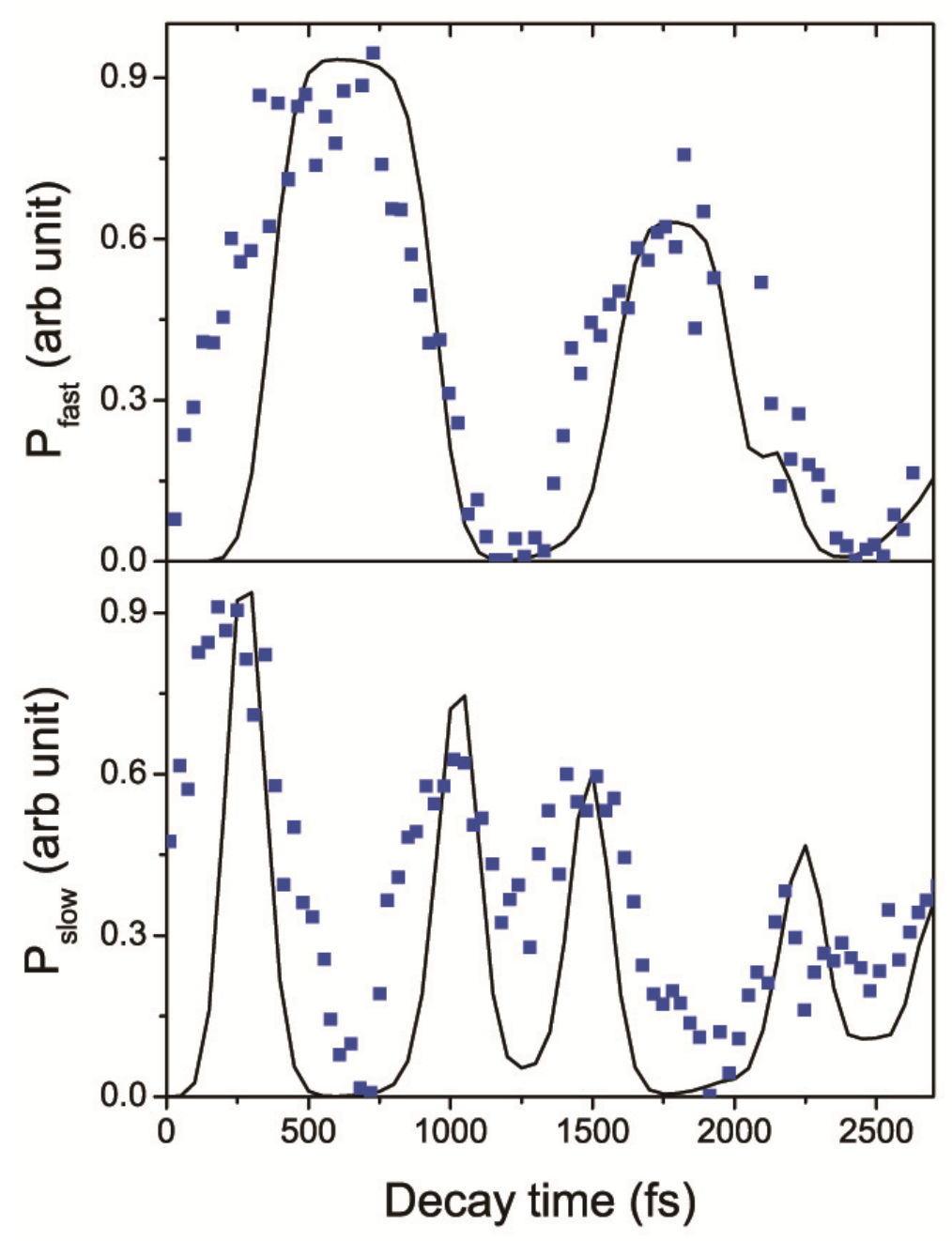

The calculated fast and slow electronic signals and their comparison with experimental results. 